\title{
The elasto-damage theory of the components assembling model
}

\author{
DENG ShouChun ${ }^{1}$, LIANG NaiGang ${ }^{1}$ \& LU HaiXing ${ }^{2}$ \\ ${ }^{1}$ Institute of Mechanics, Chinese Academy of Sciences (CAS), Beijing 100190, China \\ ${ }^{2}$ Graduate School of Ordnance Engineering College, Shijiazhuang 050003, China
}

The potential energy in materials is well approximated by pair functional which is composed of pair potentials and embedding energy. During calculating material potential energy, the orientational component and the volumetric component are derived respectively from pair potentials and embedding energy. The sum of energy of all these two kinds of components is the material potential. No matter how microstructures change, damage or fracture, at the most level, they are all the changing and breaking atomic bonds. As an abstract of atomic bonds, these components change their stiffness during damaging. Material constitutive equations have been formulated by means of assembling all components' response functions. This material model is called as the component assembling model. Theoretical analysis and numerical computing indicate that the proposed model has the capacity of reproducing some results satisfactorily, with the advantages of great conceptual simplicity, physical explicitness, and intrinsic induced anisotropy, etc.

pair functional, pair potentials, embedding energy, damage, anisotropy, orientational component, volumetric component, component assembling model

The material microstructures change under external actions $^{[1-4]}$. With loads continually increasing, microdefects such as microcracks and microvoids begin to nucleate and grow, and material mechanical properties degrade accordingly. With external loads continually increasing, these microdefects begin to join and their number increases continuously, the macrocrack emerges, and finally, material fails. The material degrading process is called damaging process, and damage occurrence and its evolution lead material mechanical performance to directional preference-anisotropy.

The process of material degrading has been explored by damage mechanics. Beginning with Kachanov ${ }^{[5]}$ proposing the conception about damage factor, damage mechanics has gotten great success in the past fifty years, and a lot of theoretical models have been proposed to handle different types of damaging, such as, LemaitreChaboche's plastic damage theory ${ }^{[2-9]}$, Kachanov's creep damage theory ${ }^{[3,5,8-10]}$, Rousselier's damage one ${ }^{[3]}$,
Gurson's model ${ }^{[6]}$, critical cavity expansion ratio ${ }^{[3]}$, Murakami-Ohno's creep damage ${ }^{[2,3,7,9]}$, Chaboche's anisotropic damage ${ }^{[6]}$, Krajcinovic's vectorial damage theory ${ }^{[4]}$, and Sidoroff's anisotropic damage theory ${ }^{[3,8]}$, etc. These theoretical models are mainly based on continuum mechanics $^{[12,13]}$ (Gurson model and critical cavity expansion ratio are based on mesomechanics). However, continuum damage theories have some drawbacks. Firstly, damage's definition isn't unique, and all these phenomenological definitions aren't physically sound. Secondly, every damage evolution equation is based on a supposed dissipation function, and the corresponding function is artificial to some extent. It's important that continuum damage formulations are very complicated, the adopted symmetrization treatment techniques are

\footnotetext{
Received March 25, 2008; accepted August 28, 2008

doi: 10.1007/s11433-009-0002-8

'Corresponding author (email: dengsc@1nm.imech.ac.cn)

Supported by the National Natural Science Foundation of China (Grant Nos.10572140 and 10232050) and the Ministry of Science and Technology Foundation (Grant No. 2002CB412706)
} 
short of physical basics, and it is particularly evident in anisotropic damage theories.

Material made up of a large number of atoms is regarded as a many-body system, and the binding forces among atoms determine the material structures and its intrinsic mechanical and electromagnetic properties ${ }^{[14-16]}$. An exact treatment of this many-body problem would require the formulation and solution of Schrodinger wave equation for all atoms considered, accounting for the interactions among the charged constituents (nuclei, electrons) and their kinetic energies. However, by using the adiabatic Born-Oppenheimer hypothesis ${ }^{[14,16]}$, the wave function is split into one part to describe the dynamics of the light electrons and the other part to describe the dynamics of the heavy nuclei. It is proper that a quasi-classical treatment of the atomic interactions and the resulting dynamics in terms of potentials and the classical equation of motion is consistent, which means the simulation of atomic configurations with instantaneously equilibrated electrons in their ground state merely requires an adequate incorporation of the forces among the atoms, expressed as derivatives of more or less empirical interatomic potential functions. The underlying potentials reflect the interaction among the atoms and are usually quantified in terms of the relative position of two or more atoms, and the parameters occurring in these potential expressions are obtained by fitting potential functions to material parameter such as, elastic constants, lattice parameter, cohesive energy, and stacking fault energy.

The potential concept enables the researchers to carry out nanoscale molecular dynamics ${ }^{[14,15,18]}$ simulations with as many as $10^{9}$ atoms. Such quantities do not allow a full mesoscale treatment of materials, which would involve about $10^{23}$ atoms. Moreover, confined by computer's capability, the computational time interval is very small. There is a long way for engineering application. Considering respectively the difficulties and merits in continuum mechanics and interatomic potentials, many researchers began to set up macroscopic constitutive models directly from interatomic potentials or combine these two theories, such as, the quasi-continuum (QC) model $^{[19,20]}$, the relations between molecular dynamics and micromorphic theories ${ }^{[21,22]}$, and the virtual internal bond (VIB) model ${ }^{[23]}$.

In this paper, the component assembling model (CAM) has been proposed and applied to damages. Po- tential energy owing to deformations has been expressed in the form of the sum of pair-functional potentials, in which, pair potentials have been computed according to discrete directions which are determined by the interactions among atoms, and the corresponding orientational component has been established, i.e., the sum of pair potentials parallel to the specified direction is the energy of the corresponding orientational component and the stiffness contribution of this part of atomic bonds is its stiffness. As a typical one-dimensional component, the orientational one bears tensile or compressive loading. Meanwhile, the other kind of component - the volumetric one has also been set up from embedding energy. For the density of electronic gas is only related to volume, the volumetric component is a typical three-dimensional one and it bears hydrostatic stress. As macroscopic phenomena of the change and break of atomic bonds, damage and fracture have been reflected by the change of stiffness and state of these components. Particularly, anisotropy has been expressed naturally by the concept of component. The constitutive equations considering damage have been formulated by assembling these two kinds of components' response functions.

\section{Devivation of CAM}

The principal view of pair-functional is that the cohesive energy of an atom is determined by the local electron density at the site, where the atom is placed ${ }^{[14-16]}$ :

$$
E_{\text {embedding }}=F(\rho) \text {, }
$$

where $F(\rho)$ is referred to as the embedding energy (function), modeling the attractive interaction as a function of the local electron density $\rho$ into where the considered atom is placed. Here, the electron density is given by

$$
\rho_{i}=\sum_{j}^{j \neq i} f\left(R_{i j}\right) .
$$

This function is interpreted as the charge at the ith nucleus owing to the spherical symmetric electronic charge densities $f(R)$ of the neighboring atoms. Hence, the function $f(R)$ evaluated at the distance $R_{i j}=$ $\left|\boldsymbol{R}_{i}-\boldsymbol{R}_{j}\right|=R^{(i, j)}, \quad(i \neq j)$ tells us exactly how much electronic density bleeds off from the site $j$ onto its neighbors, where $\boldsymbol{R}_{i}$ denotes the position of the $i$ th 
nuclear. The embedded atom method posits a total energy of the form:

$$
\begin{aligned}
& E_{\text {exact }}\left(\boldsymbol{R}_{i}, \boldsymbol{r}_{n}\right) \rightarrow E_{\text {approx }}\left[\boldsymbol{R}_{i}, \rho(\boldsymbol{r})\right] \\
= & \frac{1}{2} \sum_{i j}^{i \neq j} \phi\left(R_{i j}\right)+\sum_{i} F\left(\rho_{i}\right),
\end{aligned}
$$

where the term $\phi\left(R_{i j}\right)$ describes a pairwise isotropic interatomic potential function which is essentially repulsive and depends only on the atomic spacing $R_{i j}$ and $r_{n}$ denotes the position of the nth electron.

In practice, our interest is in excursions about the equilibrium positions, and it is convenient to define zero of energy at the initial equilibrium positions. Expanded eq. (8) by Taylor series, further progress can be made in trimming down the first term $\frac{\partial E_{\text {tot }}}{\partial \boldsymbol{R}} \cdot \delta \boldsymbol{R}$ by recognizing that the expansion is built around the equilibrium configuration, and the first term can be eliminated since at equilibrium $\frac{\partial E_{\text {tot }}}{\partial \boldsymbol{R}} \cdot \delta \boldsymbol{R} \equiv 0$. Thus, potential energy due to deformation (strain energy) is expressed in the form (the third and higher derivatives excluded):

$$
\begin{aligned}
U= & \frac{1}{4} \sum_{(\alpha, \beta)}^{\alpha \neq \beta} \phi^{\prime \prime}\left(R^{(\alpha, \beta)}\right)\left[\delta R^{(\alpha, \beta)}\right]^{2} \\
& +\frac{1}{4} \sum_{(\alpha, \beta)}^{\alpha \neq \beta} \phi^{\prime}\left(R^{(\alpha, \beta)}\right) \delta^{2} R^{(\alpha, \beta)} \\
& +\frac{1}{2} \sum_{\alpha} F^{\prime \prime}\left(\rho^{(\alpha)}\right)\left[\delta \rho^{(\alpha)}\right]^{2} \\
& +\frac{1}{2} \sum_{\alpha} F^{\prime}\left(\rho^{(\alpha)}\right) \delta^{2} \rho^{(\alpha)} .
\end{aligned}
$$

While the total of pair potentials is computed, the number of pair potentials is larger than that of atoms in the selected material element, yet the number of directions of interatomic bonds is smaller than that of atoms owing to periodic crystal configurations. Therefore, pair potentials are grouped according to directions, microstructures and their evolutions are embodied on energy changing in different directions. It is the essential idea of component assembling model. Concretely, to an appointed unit direction $\boldsymbol{m}$, an orientational component is set up accordingly. $\boldsymbol{m}$ denotes the direction of this component and the sum of pair potentials parallel to $\boldsymbol{m}$ in material is the energy of this orientational one:

$$
\begin{aligned}
E^{(\boldsymbol{m})}= & \frac{1}{4} \sum_{(\alpha, \beta)}^{\boldsymbol{R}^{(\alpha, \beta)} / / \boldsymbol{m}}\left\{\phi^{\prime \prime}\left(R^{(\alpha, \beta)}\right)\left[\delta R^{(\alpha, \beta)}\right]^{2}\right. \\
& \left.+\phi^{\prime}\left(R^{(\alpha, \beta)}\right) \delta^{2} R^{(\alpha, \beta)}\right\} .
\end{aligned}
$$

Run over all directions of the interactive atoms and set up the orientational components accordingly. Then, the change of pair potentials $U_{\mathrm{p}}$ owing to deformation is denoted by these orientational components:

$$
\begin{aligned}
U_{p} & =\frac{1}{4} \sum_{(\alpha, \beta)}^{\alpha \neq \beta}\left\{\phi^{\prime \prime}\left(R^{(\alpha, \beta)}\right)\left[\delta R^{(\alpha, \beta)}\right]^{2}+\phi^{\prime}\left(R^{(\alpha, \beta)}\right) \delta^{2} R^{(\alpha, \beta)}\right\} \\
& =\sum_{\boldsymbol{m}} E^{(\boldsymbol{m})} .
\end{aligned}
$$

Therefore, strain energy (density) is written as follows:

$$
\begin{aligned}
U= & U_{\mathrm{p}}+\frac{1}{2} \sum_{\alpha} F^{\prime \prime}\left(\rho^{(\alpha)}\right)\left[\delta \rho^{(\alpha)}\right]^{2}+\frac{1}{2} \sum_{\alpha} F^{\prime}\left(\rho^{(\alpha)}\right) \delta^{2} \rho^{(\alpha)} \\
= & \sum_{\boldsymbol{m}} E^{(\boldsymbol{m})}+\frac{1}{2} \sum_{\alpha} F^{\prime \prime}\left(\rho^{(\alpha)}\right)\left[\delta \rho^{(\alpha)}\right]^{2} \\
& +\frac{1}{2} \sum_{\alpha} F^{\prime}\left(\rho^{(\alpha)}\right) \delta^{2} \rho^{(\alpha)} .
\end{aligned}
$$

Cauchy-Born hypothesis ${ }^{[14-16,24-29]}$ builds a bridge between microscopic movements and macroscopic deformations. The hypothesis asserts that the lattice has been subjected to homogeneous deformation locally, as illustrates in Figures 1 and 2. Notes

$$
\begin{aligned}
H^{(\boldsymbol{m})}= & \frac{1}{2} \sum_{(\alpha, \beta)}^{\boldsymbol{R}^{(\alpha, \beta)} / / \boldsymbol{m}}\left\{\phi^{\prime \prime}\left(R^{(\alpha, \beta)}\right)\left[R^{(\alpha, \beta)}\right]^{2}\right. \\
& \left.-\phi^{\prime}\left(R^{(\alpha, \beta)}\right) R^{(\alpha, \beta)}\right\}=2 E^{(\boldsymbol{m})} .
\end{aligned}
$$

Substitution of eq. (8) into (7) and consideration about Cauchy-Born hypothesis, yields the expression:

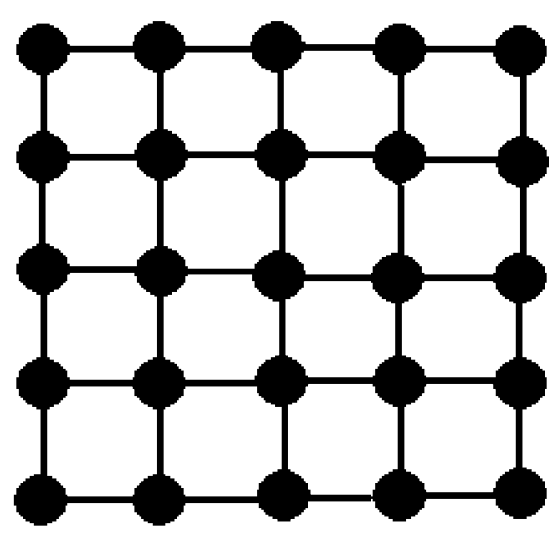

Figure 1 Un-deformed plane lattice. 


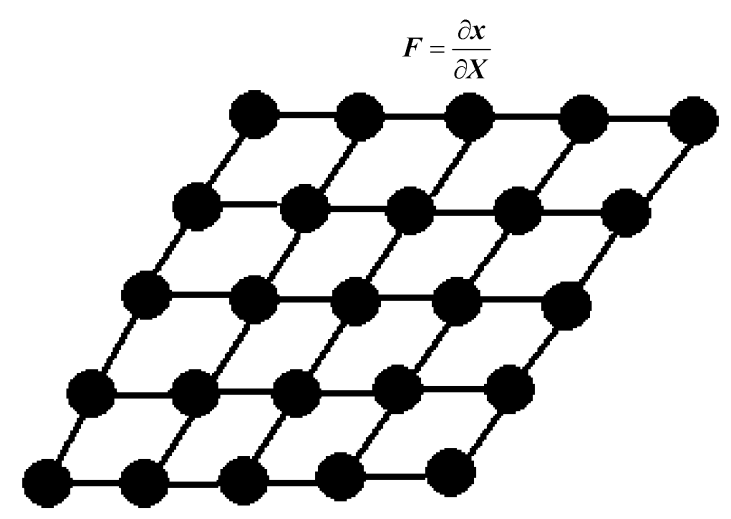

Figure 2 Deformed plane Lattice (Cauchy-Born Hypothesis).

$$
\begin{aligned}
U= & \left\{\frac{1}{2} \sum_{m} H^{(m)} m_{i} m_{\mathrm{j}} m_{k} m_{l}\right. \\
& +\left[\frac{1}{2} \sum_{\alpha} F^{\prime \prime}\left(\rho^{(\alpha)}\right)\left(\rho^{(\alpha)}\right)^{2}\right. \\
& \left.\left.+\frac{1}{2} \sum_{\alpha} F^{\prime}\left(\rho^{(\alpha)}\right) \rho^{(\alpha)}\right] \delta_{i j} \delta_{k l}\right\} \varepsilon_{i j} \varepsilon_{k l},
\end{aligned}
$$

where $m_{i}$ is the $m$ th component of vector $\boldsymbol{m}$. By energy-equivalence and comparing with continuum mechanics, yields the result:

$$
\begin{aligned}
C_{i j k l}= & \sum_{\boldsymbol{m}} H^{(\boldsymbol{m})} m_{i} m_{j} m_{k} m_{l} \\
& +\left[\sum_{\alpha} F^{\prime \prime}\left(\rho^{(\alpha)}\right)\left(\rho^{(\alpha)}\right)^{2}+\sum_{\alpha} F^{\prime}\left(\rho^{(\alpha)}\right) \rho^{(\alpha)}\right] \delta_{i j} \delta_{k l} .
\end{aligned}
$$

The first item on the right of eq. (10) is derived from pair potentials, and the last two are from embedding energy. In fact, $H^{(m)}$ is the elastic modulus of the orientational component parallel to $\boldsymbol{m}$. Similarly, the next kind of component - the volumetric one is introduced to represent the contribution of embedding energy, and its bulk modulus is given by

$$
K=\sum_{\alpha} F^{\prime \prime}\left(\rho^{(\alpha)}\right)\left(\rho^{(\alpha)}\right)^{2}-\sum_{\alpha} F^{\prime}\left(\rho^{(\alpha)}\right) \rho^{(\alpha)} .
$$

Therefore, the elasticity tensor is rewritten by pair-functional potentials, and it matches Voigt symmetry, $C_{i j k l}=C_{k l i j}=C_{i j l k}=C_{j i k l}$. Going further, eq. (10) is rewritten as follows:

$$
C_{i j k l}=\sum_{m} H^{(m)} m_{i} m_{j} m_{k} m_{l}+K \delta_{i j} \delta_{k l} .
$$

Eqs. (10) and (12) are sound for all materials that are modeled by Cauchy-Born approximation. In addition, for introducing the volumetric component, it overcomes the constraint of the Cauchy relation, $C_{i j k l}=C_{i k j l}$. Investigating eq. (10), we find that there are 16 independent components. Considering 13 independent constants in materials with one symmetric face, we deduce that CAM can be used to model many materials.

\section{CAM's parameters and comparation with other models}

\subsection{CAM's parameter calibrating}

Once the formula of pair-functional potentials is specified and the lattice configuration is known, the crystal properties can be derived directly. Actually, all materials are inhomogeneous and anisotropic, but lots of materials demonstrate macroscopic isotropy, such as most metals ${ }^{[1,15]}$. In particular, for homogeneous materials (the observing length is much larger than the characteristic length), the directions between nucleus spread all over the space, and the electron density is treated as a constant, that is, it admits the representation:

$$
\begin{aligned}
& H(\theta, \varphi) \Delta \Omega=H(\theta, \varphi) \sin \varphi \Delta \theta \Delta \varphi \\
& =\frac{1}{2} \sum_{(\alpha, \beta)}^{\boldsymbol{R}^{(\alpha, \beta)} \in \Delta \Omega}\left\{\phi^{\prime \prime}\left(R^{(\alpha, \beta)}\right)\left(R^{(\alpha, \beta)}\right)^{2}-\phi^{\prime}\left(R^{(\alpha, \beta)}\right) R^{(\alpha, \beta)}\right\},
\end{aligned}
$$

where $\Delta \Omega$ is a little solid angle around the direction $(\theta, \varphi)$, and

$$
K(\theta, \varphi)=\sum_{\alpha} F^{\prime \prime}\left(\rho^{(\alpha)}\right)\left(\rho^{(\alpha)}\right)^{2}+\sum_{\alpha} F^{\prime}\left(\rho^{(\alpha)}\right) \rho^{(\alpha)} .
$$

Because the changes of electronic density do only relate to volumetric strain, and infinitesimal deformation is considered, thus eq. (14) becomes

$$
K(\theta, \varphi)=K \equiv \text { const. }
$$

The summation form in eq. (10) or (14) is rewritten in an integral form

$$
\begin{aligned}
C_{i j k l}= & \int_{0}^{\frac{\pi}{2}} \int_{0}^{\pi} H(\theta, \varphi) m_{i}(\theta, \varphi) m_{j}(\theta, \varphi) m_{k}(\theta, \varphi) m_{l}(\theta, \varphi) \\
& \times \sin \theta \mathrm{d} \theta \mathrm{d} \varphi+K \delta_{i j} \delta_{k l} .
\end{aligned}
$$

The above equation is the constitutive equation for nomogeneous materials. Conveniently, the integral of eq. (16) would be transformed to discrete summation for numerical computing, and the selected directions turned into the orientational components' directions. After analyzing and computing, we have found that 12 representative directions for planar and 46 for spatial are 
enough, as displayed representatively in Figures 3 and 4. The discretized orientational component's stiffness is expressed as

$$
\begin{aligned}
& H(\theta, \varphi) \Delta \Omega=H(\theta, \varphi) \sin \varphi \Delta \theta \Delta \varphi \\
& =\frac{1}{2} \sum_{(\alpha, \beta)}^{\boldsymbol{R}^{(\alpha, \beta)} \in \Delta \Omega}\left\{\phi^{\prime \prime}\left(R^{(\alpha, \beta)}\right)\left(R^{(\alpha, \beta)}\right)^{2}-\phi^{\prime}\left(R^{(\alpha, \beta)}\right) R^{(\alpha, \beta)}\right\},
\end{aligned}
$$

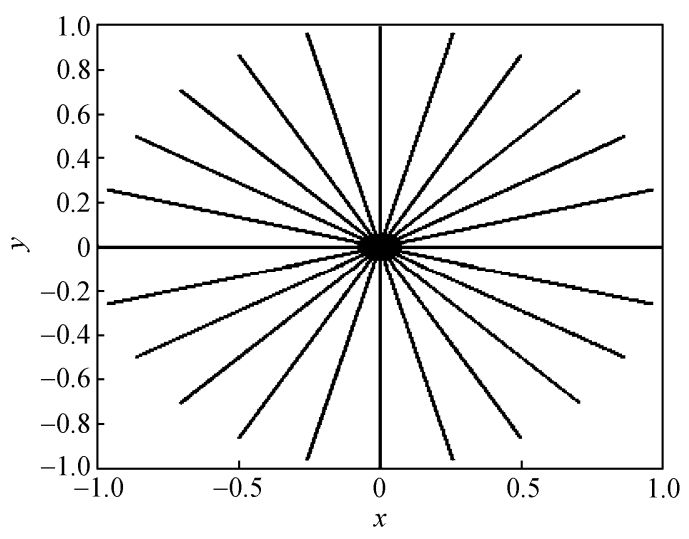

Figure 3 Planar discrete orientational components.

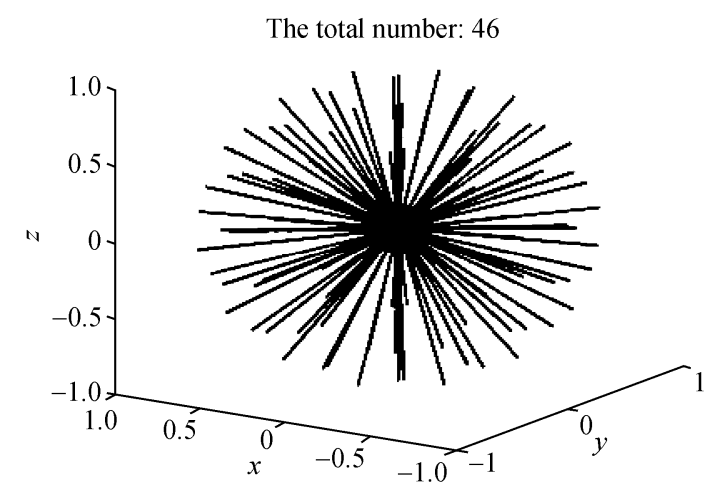

Figure 4 Spatial discrete orientational components.

Material constitutive equation has the same form as eq. (10) or (12). In particular, for homogeneous and isotropic materials, there stands

$$
H(\theta, \varphi)=H=\text { const. }
$$

Integrating eq. (16) on the up half unit sphere yields

$$
C_{i j k l}=\left(\frac{2 \pi}{15} H+K\right) \delta_{i j} \delta_{k l}+\frac{2 \pi}{15} H\left(\delta_{i k} \delta_{j l}+\delta_{i l} \delta_{j k}\right) \text {. }
$$

Comparing with traditional continuum mechan$\operatorname{ics}^{[12,13]}$, yields the result:

$$
\left\{\begin{array}{l}
H=\frac{15}{2 \pi} \mu, \\
K=\lambda-\mu,
\end{array}\right.
$$

where $\lambda$ and $\mu$ denote Lame's coefficients.
Here it needs to be stressed that the adopted CauchyBorn rule is limited, but it can be overcome if we employ the modified Cauchy-Born hypothesis ${ }^{[24-29]}$, such as the integral mean value based ${ }^{[24]}$ and high-order ${ }^{[26]}$ Cauchy-Born hypothesis, which have succeeded in describing inhomogeneous deformation in quasicontinuum model, such as dislocation dynamics, plastic slip and nano-indentation ${ }^{[19,20]}$. The exponent Cauchy-Born hypothesis $^{[29]}$ has been used in shape-distortion of the thin-wall carbon nanotubes. The Cauchy-Born hypothesis which considers temperature's effect ${ }^{[29]}$ is used in temperature changing significantly. It's worth noting that Chen et al. ${ }^{[21,22]}$ proved that molecular dynamics was related to continuum theory if atom's deformation macroscopically obeyed deformation gradient in statistical average.

\subsection{Comparation with other models}

QC, VIB and the component assembling model have the same physical foundation, and they are all from interatomic potentials. In QC, the atoms are grouped according to the quantities of interatomic potentials, by combining with continuum mechanics (QC is used near crack tips or in slip bands and continuum mechanics is used in other regions). In component assembling model, the atoms are grouped according to the directions. VIB model is derived from pair potentials and has a simple form. In which, owing to not considering the embedding energy, it is constrained by the Cauchy relation, $C_{i j k l}=$ $C_{i k j l}$.

Just like microplane model ${ }^{[30-33]}$, the basic research element is not RVE. The basic element in microplane model is discrete microplanes, and the stress-strain relations are defined independently of planes of all possible orientations in the microstructure, regardles of elasticity, plasticity and damage. Moreover, the microplane stresses or strains are constrained kinematically or statically to the macroscopic stress or strain tensors in a weak sense. There are three basic research elements in the component assembling model, and they are respectively the orientational component, the volumetric and slip ones. Actually, these components are re-divisions of RVE according to different deformation mechanisms, in which, as a $1 \mathrm{D}$ bar, the orientational component only bears the tensile or compressive loads. As a typical 2D component, the slip one bears shear loads. The 3D volumetric component bears the hydrostatic stresses. 
Material is treated as a component assembly, and its constitutive equations have been formulated by assembling all kinds of components' response functions. Anisotropy has been incorporated naturally via component concept. Meanwhile, material damage and yield have been reflected via different components.

Cohesive zone model ${ }^{[34-37]}$ has been used to simulate fracture process extensively. Material fracture work is expressed by cohesive zone models constitutive. Actually, material damage and fracture processes are atomistic bonds change and continuous break, and the cohesive zone models constitutive can be gotten from interatomic potentials. Cohesive zone model constitution is gotten from components response functions by means of energy equivalence. Actually, material damage and its microstructure evolutions occur in space but not plane.

\section{Quasi-brittle damage}

Fracture of engineering structures is often preceded by considerable changes in the microstructures of the material they are made of. Examples are microcracking in concrete, fibre pull-out or delamination in composites and the formation of voids in ductile metals. Accurate failure predictions can only be obtained if this microstructural damage is taken into account in the fracture modeling. This requirement has led to the development of so-called local or continuum approaches to fracture, in which fracture is regarded as the ultimate consequence of the material degradation process. In these methods, the degradation is often modeled by using continuum damage mechanics. Continuum damage theories introduce a set of field variables (damage variables) which explicitly describe the local loss of material integrity. A crack is represented by the part of the material domain in which the damage has become critical, where the material cannot sustain stress anymore. Redistribution of stresses results in the concentration of deformation and damage growth in a relatively small region in front of crack tip. It is the growth of damage in this process zone which determines in which direction and at which rate the crack will propagate. Crack initiation and growth thus follow naturally from the standard continuum theory, instead of from separate fracture criteria.

It is true that, on though microstructures change and damage and fracture, they are all the changing and break of atomic bonds. The orientational component is an abstract of atomic bonds. When atomic debonding emerges, the corresponding orientational component changes their mechanical properties, such as stiffness. The more atomic debonding occurs, the more stiffness changes:

$$
D=1-\frac{H}{H_{0}},
$$

where $H_{0}$ and $H$ denote respectively the initial and instantaneous (damaged) secant stiffness of the component, $D$ its damage factor and $0 \leqslant D \leqslant 1$. A scalar value is enough for a 1-D component, it is a microscopic value (however, for material element, its constitution is a typical fourth order tensor, as eq. (16) shows). Meantime, as a typical 1-D component, the orientational one has simple constitutive relation, and it is expressed as follows:

$$
\sigma_{m}=H \varepsilon_{m}=(1-D) H_{0} \varepsilon_{m},
$$

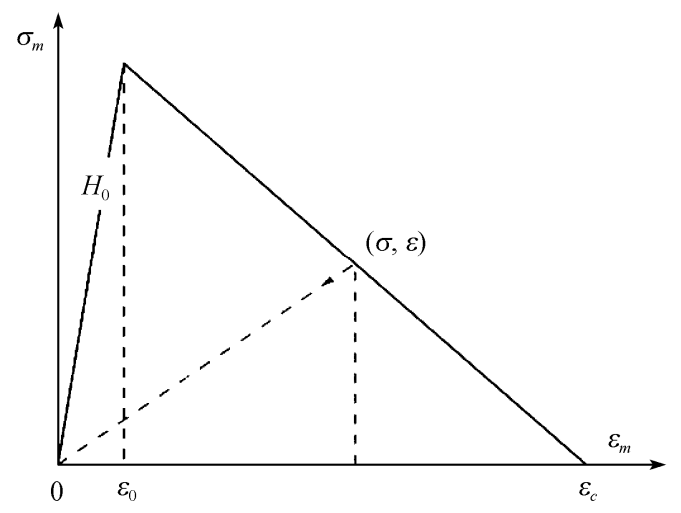

Figure 5 Configuration of the orientational component's damage.

where $\sigma_{m}$ denotes the stress of the component $\boldsymbol{m}$ and $\varepsilon_{m}$ is strain. There is

$$
\varepsilon_{m}=\varepsilon_{i j} m_{i} m_{j}
$$

where $\varepsilon_{i j}$ denotes the strain. Without loss of generality, we consider tension-induced damage as well as compression-induced damage but no same damage evolution laws. For 1-D simple orientational component, its damage factor must be the function of the maximum strain and / or the minimum strain, damage thresholds are denoted as $\varepsilon_{+\mathrm{cr}}$ and $\varepsilon_{-\mathrm{cr}}$ which change along with damage, and then, damage evolution equation has the following form:

$$
D=D_{1}\left(\varepsilon_{+\mathrm{cr}}\right)=D_{2}\left(\varepsilon_{-\mathrm{cr}}\right) .
$$

To the whole deformation history $\varepsilon_{i j}(t)$, there are $\varepsilon_{+\mathrm{cr}}=\max \left(\varepsilon_{m}(t)\right)=\max \left(\varepsilon_{i j}(t) m_{i} m_{j}\right) \quad$ and $\quad \varepsilon_{-\mathrm{cr}}=$ 
$\min \left(\varepsilon_{m}(t)\right)=\min \left(\varepsilon_{i j}(t) m_{i} m_{j}\right)$. Therewith, the orientational component's damage is expressed as

$$
D=D\left(\boldsymbol{Q}, \boldsymbol{\varepsilon}^{(\boldsymbol{m})}\right)
$$

where $Q=\boldsymbol{m} \otimes \boldsymbol{m}$ denotes the kinetic tensor, and $\varepsilon^{(m)}$ the corresponding orientational component's eigen-strain (internal variable). Different components have different $\boldsymbol{\varepsilon}^{(\boldsymbol{m})}$, yet there always has $\boldsymbol{Q}: \boldsymbol{\varepsilon}^{(\boldsymbol{m})}=\varepsilon_{+\mathrm{cr}}$ $\left(=\varepsilon_{\text {-cr }}\right)$. Correspondingly, damage condition is

$$
f\left(\varepsilon_{m}, \varepsilon_{\mathrm{cr}}\right)=\left\{\begin{array}{l}
\varepsilon_{m}-\varepsilon_{+\mathrm{cr}} \leqslant 0, \text { tension, } \\
\varepsilon_{-\mathrm{cr}}-\varepsilon_{m} \leqslant 0, \text { compression. }
\end{array}\right.
$$

Damage threshold has always the same symbol with its current strain, $\varepsilon_{\mathrm{cr}}=\varepsilon_{+\mathrm{cr}}$ and $\varepsilon_{\mathrm{cr}}=\varepsilon_{-\mathrm{cr}}$. In addition, there is Kuhn-Tucker condition:

$$
\dot{D} \geqslant 0, \quad f\left(\varepsilon_{m}, \varepsilon_{\text {cr }}\right) \leqslant 0, \quad \dot{D} f\left(\varepsilon_{m}, \varepsilon_{\text {cr }}\right)=0 .
$$

If crack closure effect is excludable, damage threshold evolution is given as

$$
\dot{D}=\frac{\mathrm{d} D}{\mathrm{~d} \varepsilon_{\mathrm{cr}}} \dot{\varepsilon}_{\mathrm{cr}}
$$

Damage's consistence condition is

$$
\dot{D} \dot{f}\left(\varepsilon_{m}, \varepsilon_{\mathrm{cr}}\right)=0 \text {, when } f\left(\varepsilon_{m}, \varepsilon_{\mathrm{cr}}\right)=0 .
$$

For infinitesimal displacement, differentiating eq. (21) and considering eq. (22), there is

$$
\begin{aligned}
\dot{\sigma}_{m} & =(1-D) H_{0} m_{i} m_{j} \dot{\varepsilon}_{i j}-H_{0} \varepsilon_{m} \dot{D} \\
& =\left\{(1-D) H_{0}-H_{0} \varepsilon_{m} \frac{\mathrm{d} D}{\mathrm{~d} \varepsilon_{\mathrm{cr}}}\right\} m_{i} m_{j} \dot{\varepsilon}_{i j} .
\end{aligned}
$$

The stress contribution of the single orientational component to the whole stress field is given as

$$
\sigma_{i j}=\sigma_{m} m_{i} m_{j},
$$

The stiffness of volumetric componentis relates to volume changes and its change is omitted for infinitesimal strain. Assembly all components, then get the rate form of constitutive relations:

$$
\begin{aligned}
\dot{\sigma}_{i j}= & \left\{\sum_{s=1}^{M}\left[\left(1-D^{s}\right) H_{0}^{s}-H_{0}^{s} \varepsilon_{m}^{s}\left(\frac{\mathrm{d} D}{\mathrm{~d} \varepsilon_{\mathrm{cr}}}\right)^{s}\right] m_{i}^{s} m_{j}^{s} m_{k}^{s} m_{l}^{s}\right. \\
& \left.+\mathrm{K} \delta_{i j} \delta_{k l}\right\} \dot{\varepsilon}_{k l} .
\end{aligned}
$$

Correspondingly, its quasi-brittle tangent tensor is

$$
C_{i j k l}^{\mathrm{ed}}=\left\{\sum_{s=1}^{M}\left[\left(1-D^{s}\right) H_{0}^{s}-H_{0}^{s} \varepsilon_{m}^{s}\left(\frac{\mathrm{d} D}{\mathrm{~d} \varepsilon_{\mathrm{cr}}}\right)^{s}\right] m_{i}^{s} m_{j}^{s} m_{k}^{s} m_{l}^{s}\right.
$$

$$
\left.+K \delta_{i j} \delta_{k l}\right\}
$$

in the above two equations, and damage induced stiffness is $H_{0} \varepsilon_{m}\left(\frac{\mathrm{d} D}{\mathrm{~d} \varepsilon_{\mathrm{cr}}}\right)$, which only occurs as damage continuingly. Here, it is stressed that damage at material representative volume element (RVE) is anisotropic though every component's damage is a scalar. Additionally, eq. (32) also describes component inconsistent damage evolution laws, and it implies that $\left(\frac{\mathrm{d} D}{\mathrm{~d} \varepsilon_{\mathrm{cr}}}\right)$ is different.

The aforementioned just considers the orientational components' damage, and the stiffness change of volumetric component in-negligible as displacement is comparatively large. Embedding potential is essentially Coulomb's potential, and it is proportional to electric charge. As volume increasing and electron density decreasing, the volumetric component's stiffness decreases correspondingly and vice versa.

$$
K=K\left(\varepsilon_{i i}\right) .
$$

Elastic damage stiffness tensor is rewritten as

$$
\begin{aligned}
C_{i j k l}^{\mathrm{ed}}= & \left\{\sum_{s=1}^{M}\left[\left(1-D^{s}\right) H_{0}^{s}-H_{0}^{s} \varepsilon_{n}^{s}\left(\frac{\mathrm{d} D}{\mathrm{~d} \varepsilon_{\mathrm{cr}}}\right)^{s}\right] m_{i}^{s} m_{j}^{s} m_{k}^{s} m_{l}^{s}\right. \\
& \left.+\left(K+\varepsilon_{q q} \frac{\partial K}{\partial \varepsilon_{p p}}\right) \delta_{i j} \delta_{k l}\right\}
\end{aligned}
$$

The above just considers the quasi-unilateral condition. Actually, it is easy to consider the crack closure for the orientational component has only two statestension and compression. Denotes orientational component closure coefficient:

$$
\langle\alpha\rangle=\left\{\begin{array}{l}
1, \varepsilon \geqslant 0, \\
\alpha, \varepsilon<0,
\end{array}\right.
$$

and eq. (32) changes as follows:

$$
\begin{aligned}
C_{i j k l}^{\mathrm{ed}}= & \left\{\sum_{s=1}^{M}\left[\left(1-\left\langle\alpha^{s}\right\rangle D^{s}\right) H_{0}^{s}-\left\langle\alpha^{s}\right\rangle H_{0}^{s} \varepsilon_{n}^{s}\left(\frac{\mathrm{d} D}{\mathrm{~d} \varepsilon_{\mathrm{cr}}}\right)^{s}\right]\right. \\
& \left.\times m_{i}^{s} m_{j}^{s} m_{k}^{s} m_{l}^{s}+\left(K+\varepsilon_{q q} \frac{\partial K}{\partial \varepsilon_{p p}}\right) \delta_{i j} \delta_{k l}\right\} .
\end{aligned}
$$




\section{Numerical method and examples}

The equations of equilibrium is

$$
\sigma_{i j, j}+F_{i}=0
$$

where $\boldsymbol{F}$ denotes external force. The appropriate boundary conditions are exerted during solving by using FEM:

$$
\left\{\begin{array}{l}
\boldsymbol{u}=\boldsymbol{u}^{b}, \boldsymbol{u} \in \Gamma_{u}, \\
\boldsymbol{t}=\boldsymbol{\sigma} \cdot \boldsymbol{n}, \boldsymbol{t} \in \Gamma_{\sigma},
\end{array}\right.
$$

We do weighted $\boldsymbol{S}$ integrate at whole domain $\Omega$, and get the weak form of control equations:

$$
\int_{\Omega} S_{i}\left(\sigma_{i j, j}+F_{i}\right) \mathrm{d} \Omega=0 .
$$

Integrating by parts, utilizing divergence theorem and considering boundary conditions (eq. (38)), there is

$$
\int_{\Omega} S_{i, j} \sigma_{i j} \mathrm{~d} \Omega=\int_{\Omega} S_{i} F_{i} \mathrm{~d} \Omega+\int_{\Gamma} S_{i} t_{i} \mathrm{~d} \Gamma .
$$

Displacement field $\boldsymbol{u}$ is discretized in the following:

$$
\left\{\begin{array}{l}
u_{i}=N_{i j} d_{j}, \\
u_{i, k}=N_{i j, k} d_{j},
\end{array}\right.
$$

where $\boldsymbol{d}$ is nodal displacement vector, and $\boldsymbol{N}$ shape function. According to the Bubnov-Galerkin method ${ }^{[33]}$, the corresponding weighted function is discretized as follows:

$$
\left\{\begin{array}{l}
S_{i}=N_{i j} d_{j}^{s}, \\
S_{i, k}=N_{i j, k} d_{j}^{s},
\end{array}\right.
$$

The ultimate equations must satisfy all nodal permissible displacement $\boldsymbol{d}^{s}$. Concretely, there is

$$
\int_{\Omega} N_{i j, k} \sigma_{i k} \mathrm{~d} \Omega=\int_{\Omega} N_{i j} F_{i} \mathrm{~d} \Omega+\int_{\Gamma} N_{i j} t_{i} \mathrm{~d} \Gamma .
$$

In order to construct consistent incrementaliterative process, we have to linearize eq.(31). For iterative step $i$, the nodal displacement is rewritten in the form:

$$
d_{j}^{i}=d_{j}^{i-1}+\Delta d_{j},
$$

which leads to the stress at an integral point having the form:

$$
\sigma_{k l}^{i}=\sigma_{k l}^{i-1}+\Delta \sigma_{k l}
$$

Similarly, there are

$$
\left\{\begin{array}{l}
\varepsilon_{s t}^{i}=\varepsilon_{s t}^{i-1}+\Delta \varepsilon_{s t}, \\
\Delta \varepsilon_{s t}=\frac{1}{2}\left(N_{s j, t}+N_{t j, s}\right) \Delta d_{j} .
\end{array}\right.
$$

It is pointed out that the superscript $i$ implies incremental or iterative step and subscript $i$ the component of tensor. Considering the above three equations and (31), we have the following result:

$$
\begin{aligned}
& \frac{1}{2} \int_{\Omega} N_{i j, k} C_{i k s t}^{e d}\left(N_{s p, t}+N_{t p, s}\right) \Delta d_{p} \mathrm{~d} \Omega \\
= & \int_{\Omega} N_{i j} F_{i} \mathrm{~d} \Omega+\int_{\Gamma} N_{i j} t_{i} \mathrm{~d} \Gamma-\int_{\Omega} N_{i j, k} \sigma_{i k}^{i-1} \mathrm{~d} \Omega .
\end{aligned}
$$

Rewrite eq. (42) into matrix form:

$$
[K]\{\Delta d\}=\left\{F^{\mathrm{ext}}\right\}-\left\{F^{\mathrm{in}, i-1}\right\}=\{R\},
$$

where $\{R\}$ denotes the nodal residual force, and the other matrixes and column vectors are defined as follows:

$$
\begin{aligned}
& K_{j p}=\frac{1}{2} \int_{\Omega} N_{i j, k} C_{i k s t}^{\mathrm{ed}}\left(N_{s p, t}+N_{t p, s}\right) \mathrm{d} \Omega, \\
& F_{j}^{\mathrm{ext}}=\int_{\Omega} N_{i j} F_{i} \mathrm{~d} \Omega+\int_{\Gamma} N_{i j} t_{i} \mathrm{~d} \Gamma, \\
& F_{j}^{\mathrm{in}, i-1}=\int_{\Omega} N_{i j, k} \sigma_{i k}^{i-1} \mathrm{~d} \Omega .
\end{aligned}
$$

We calibrate orientational component's response function by fitting experimental data. Figure 6 illustrates the unit length element under monodirectional stretching, and Figures (7a) and (7b) are the corresponding stress-strain and response function curves, in which, Young's modulus is $E=21400 \mathrm{GPa}$ and Poisson's ratio $v=0.2$. Only the orientational component's tension-induced damage is considered, and its spatial distribution is illustrated in Figure 4. The initial stiffnesses of orientational and volumetric components are respectively $H_{0}=\frac{15}{2 \pi} \frac{E}{2(1+v)} \frac{2 \pi}{46} \doteq 2907.6 \mathrm{MPa}$ and $K \doteq$ $-4458.3 \mathrm{MPa}$.

The axial damaged Young's modulus $\tilde{E}$ is defined by axial tension:

$$
\begin{aligned}
F & =\sigma_{11} A \\
& =A\left[\sum_{\alpha=1}^{M}\left(1-D^{(\alpha)}\right) H_{0}^{(\alpha)} m_{1}^{(\alpha)} m_{1}^{(\alpha)} m_{k}^{(\alpha)} m_{l}^{(\alpha)}+K \delta_{k l}\right] \varepsilon_{k l} \\
& \approx \tilde{E} \varepsilon_{11} A .
\end{aligned}
$$

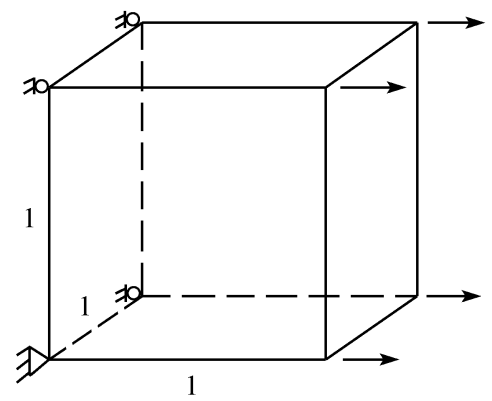

Figure 6 Configuration of element and its boundary conditions. 

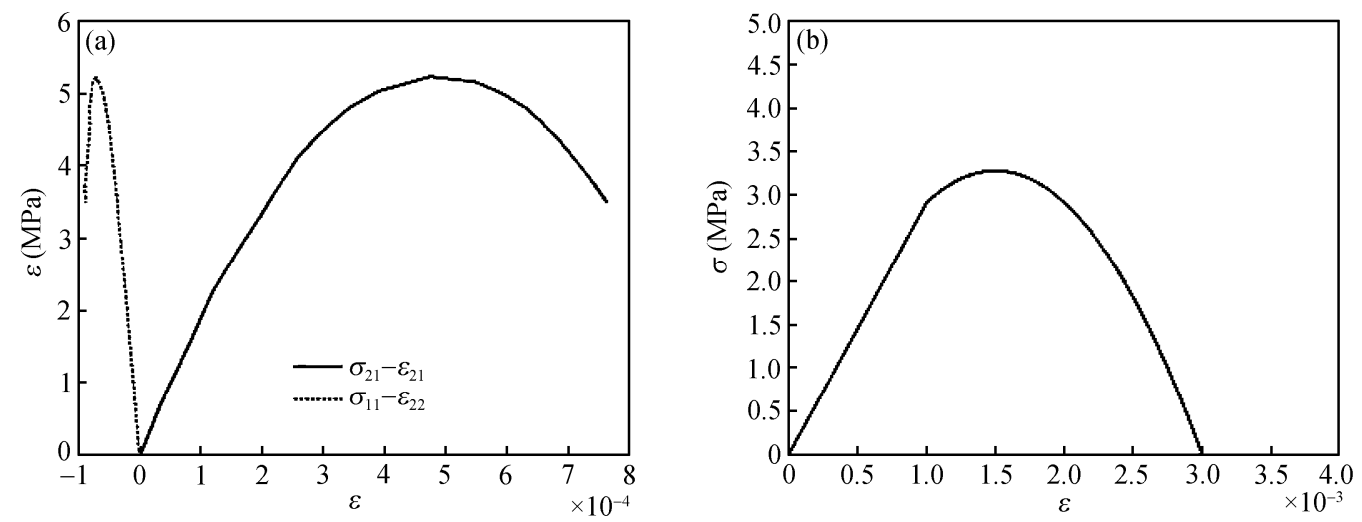

Figure 7 (a) The stress-strain curve ${ }^{[106]}$; (b) The response function.

then,

$$
\begin{aligned}
\tilde{E} & \approx \frac{F}{A \varepsilon_{11}}=\frac{\sigma_{11}}{\varepsilon_{11}} \\
& =\left[\sum_{\alpha=1}^{M}\left(1-D^{(\alpha)}\right) H_{0}^{(\alpha)} m_{1}^{(\alpha)} m_{1}^{(\alpha)} m_{k}^{(\alpha)} m_{l}^{(\alpha)}+K \delta_{k l}\right] \frac{\varepsilon_{k l}}{\varepsilon_{11}} .
\end{aligned}
$$

In a similar way, the changing Poisson's ratio $\tilde{v}$ is expressed as

$$
\tilde{v}=\left|\frac{\varepsilon_{22}}{\varepsilon_{11}}\right|=-\frac{S_{2211}^{\text {secant }}}{S_{1111}^{\text {secant }}} .
$$

Figure 8 is the equivalent transverse Poisson's ratioaxial strain curve.

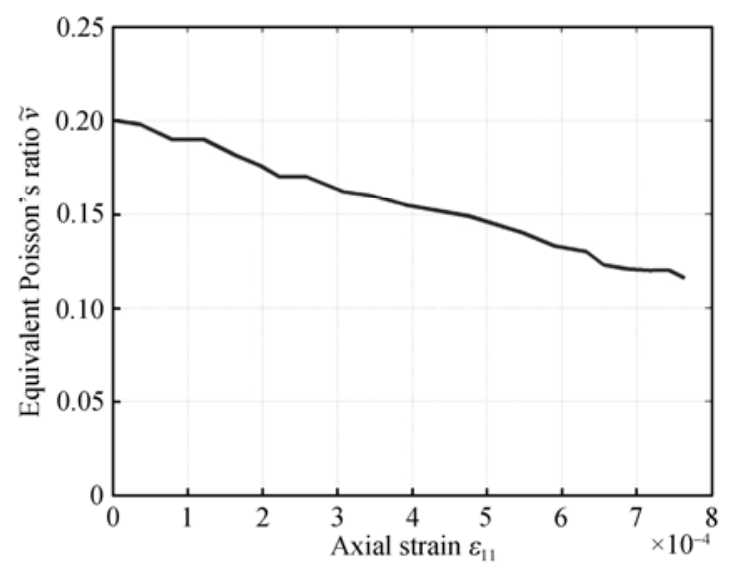

Figure 8 Equivalent transverse Poisso's ratio $\tilde{v}$ vs. axial strain $\varepsilon_{11}$.

Model's validity is proven by comparing with experiments. The wedge equipment and its assembling are illustrated in Figure 9, and specimen is illustrated in Figure 10. Specimen thickness $t=97 \mathrm{~mm}$. Material initial modulus is $E=12400 \mathrm{MPa}$, and Poisson's ratio $v=$ 0.3. 46 Conponents' spatial distribution is the same as displayed in Figure 4. The initial stiffnesses of orientational and volumetric components are

$$
\left\{\begin{array}{l}
H=\frac{15}{2 \pi} \mu \cdot \frac{2 \pi}{46}=\frac{15 E}{92(1+v)} \doteq 1555.2 \mathrm{MPa}, \\
K=\lambda-\mu=\frac{(4 v-1) E}{2(1+v)(1-2 v)} \doteq 2384.6 \mathrm{MPa} .
\end{array}\right.
$$

The stiffness change of volumetric component can be ignored under small deformation. Compressive strength of concrete is much larger than its tensile strength, and it is rational to only consider compressive damage.

Figure 11 illustrates the component strain-stress relation, in which its constitution is approximated respectively by two-line and three-line. Figures 12 and 13 are the load-displacement curves according to different constitutive relations. Compared with experimental results (indicated by discrete circles), three-line constitution

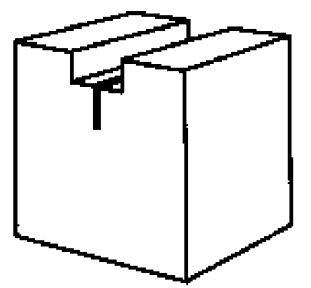

(a)

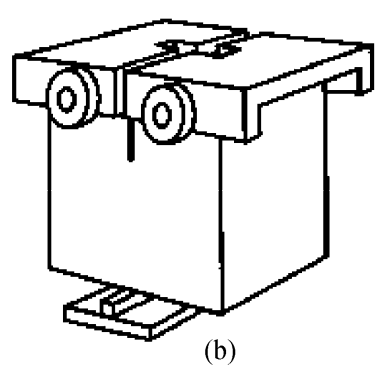

(b)

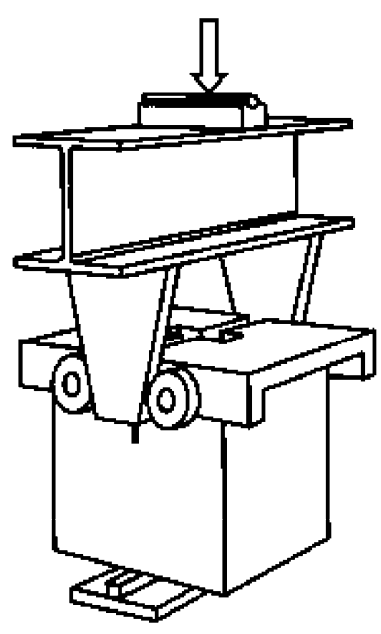

(c)
Figure 9 Configuration of wedge instrument ${ }^{[34]}$. 


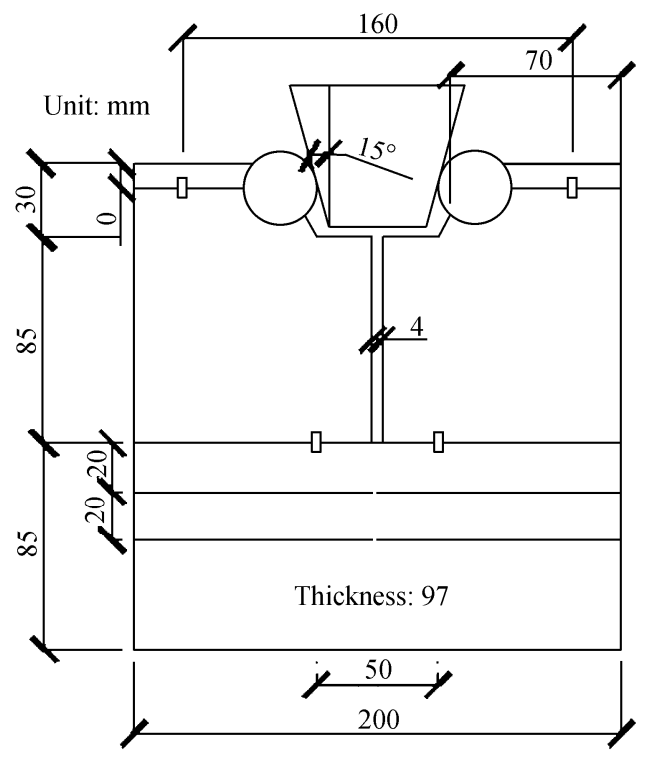

Figure 10 The geometrical configuration of concrete specimen ${ }^{[34]}$.

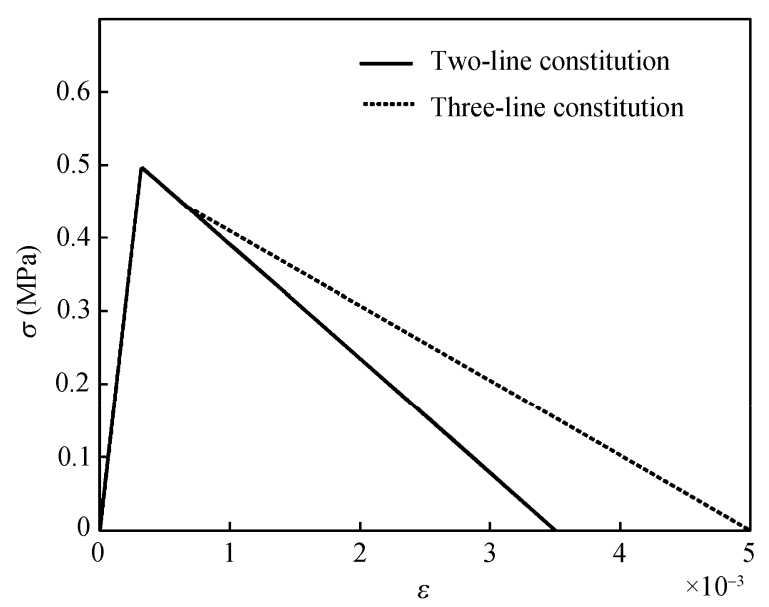

Figure 11 Orientational component's constitutive curves.

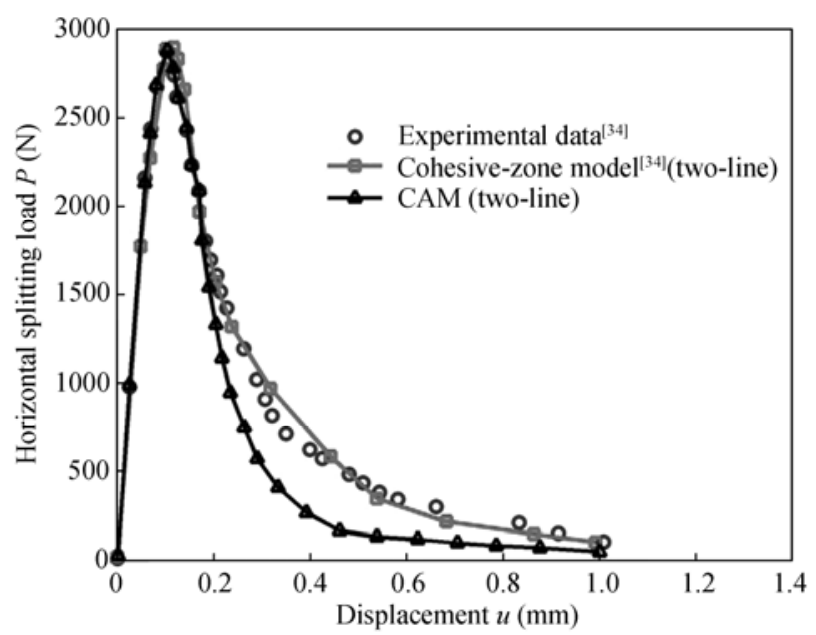

Figure 12 The numerical results of two-line constitutive.

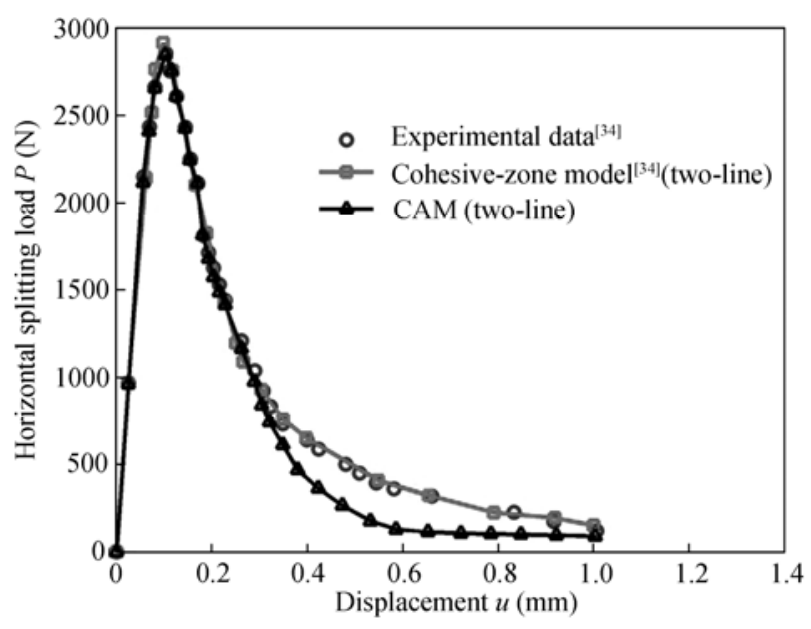

Figure 13 The numerical results of three-line constitutive.

matches better, and it indicates that we can get better result if we adopt more complicated constitution. The numerical results by using "cohesive-zone" $\operatorname{model}^{[34]}$ have also been presented in Figures 12 and 13. It is obvious that CAM and cohesive-zone model have high precision and predict peak loads accurately. After Past peak value, the results by CAM decline allegretto.

\section{Conclusion}

CAM has been set up based on pair functional, in which, two kinds of components - the orientational and volumetric ones have been derived respectively from pair potentials and embedding energy. As a typical 1-D component, the orientational component can only bear tensile or compressive load. However, the volumetric component is a typical 3-D one and it can only bear hydrostatic pressure. Material has been treated as a component assembly, and its constitutive equations have been formed by assembling all components' response functions. Theoretical analysis indicates that the proposed method has the capacity of re-expressing the generalized Hooke's Law (eq. (3)) and reproducing some results satisfactorily.

The proposed model has been developed for quasibrittle damage, in which, anisotropy and its evolution have been incorporated appropriately via the component concept. Theoretical and preliminary numerical computon indicates that the proposed model has the capacity of re-expressing the generalized Hooke's Law and reproducing some results satisfactorily, with the advantages of great conceptual simplicity, physical explicit- 
ness ${ }^{[38-41]}$, and intrinsic induced anisotropy, etc. In addition, the proposed model has the capacity of eliminating

1 Lai Z L. Metal Crystal Defects and Its Mechanical Characteristic (in Chinese). Beijing: Metallurgical Industry Press, 1988

2 Lou Z W. Foundation of Damage Mechanics (in Chinese). Xi'an: Xi'an Jiaotong University Press, 1991

3 Yu S W, Feng X Q. Dmage Mechanics (in Chinese). Qinghua: Qinghua University Press, 1997

4 Krajcinovic D. Damage Mechanics. Amsterdam: Elsevier, 1996

5 Kachanov L M. On the time to failure under creep condition. Izv Akad Nauk USSR Otd Tekhn Nauk, 1958, 8: 26-31

6 Chaboche J L. Continuum damage mechanics: Part I general concepts, and Part II damage growth, crack initiation, and crack growth. J Appl Mech, 1988, 55: 59-72

7 Lemaitre J. Local approach of fracture. Eng Fract Mech, 1986, 25(5/6): $523-537$

8 Lemaitre J. Damage Mechanics Tutorial (in Chinese). Beijing: Academic Press, 1996

9 Lemaitre J, Desmorat R. Engineering Damage Mechanics: Ductile, Creep, Fatigue and Brittle Failures. Berlin: Springer, 2005

10 Kachanov L M. Introduction to Continuum Damage Mechanics. Dordrecht: Martinus Nijhof Publishers, 1986

11 Reusch F, Svendsen B, Klingbeil D. A non-local extension of Gurson-based ductile damage modeling. Comput Mater Sci, 2003, 26: $219-229$

12 Kuang Z B. Nonlinear Continuum Mechanics (in Chinese). Shanghai: Shanghai Jiaotong University Press, 2002

13 Feng Y C. Foundations of Solid Mechanics. New Jersey: Prentice Hall, 1965

14 Raabe D. Computational Materials Science-The Simulation of Materials Microstructures and Properties. Weinheim: WILEY-VCH, 1998

15 Raabe D, Roters F, Barlet F, et al. Continuum Scale Simulation of Engineering Materials: Fundamentals-Microstructures-Process Applications. Weinheim: WILEY-VCH Verlag GmbH \& Co KGaA, 2004

16 Phillips R. Crystals Defects and Microstructures-Modeling Across Scales. Cambridge: Cambridge University Press, 2001

17 Landau L D, Lifshits E M. Quantum Mechanics: Non-relativistic Theory. Oxford: Pergamon Press, 1977

18 Frank, Smidt. Molecular Simulation: From Algorithm to Application (in Chinese). Beijing: Chemical Industry Press, 2002

19 Miller R, Ortiz M, Phillips R, et al. Quasicontinuum models of fracture and plasticity. Eng Fract Mech, 1998, 61: 427-444

20 Miller R, Tadmor E B, Phillips R, et al. Quasicontinuum simulation of fracture at the atomic scale. Model Simul Mater Sci Eng, 1998, 6: 607-638

21 Chen Y P, James D L, Eskandarian A. Atomistic viewpoint of the applicability of microcontinuum theories. Int J Solid Struct, 2004, 41: $2085-2097$ mesh sensitivity just as non-local ${ }^{[42-44]}$, strain gradient ${ }^{[33,42,44-48]}$ and Cosserat ${ }^{[49,50]}$ models do.

22 Chen Y P, James D L. Connecting molecular dynamics to micromorphic theory (I, II). Physica A, 2003, 322: 359-392

23 Gao H J, Klein P J. Mech Phys Solids, 1998, 46(2): 187-218

24 Chandraseker K, Mukherjee S. Modifications to the Cauchy-Born Rule. Applications in the Deformation of single-walled Carbon Nanotubes. Int J Solids Struct, 2006, 43: 7128-7144

25 Ericksen J L. The Cauchy and Born hypotheses for crystals. In: Gurtin M E, ed. Phase Transformations and Material Instabilities in Solids. New York: Academic Press, 1984. 61-77

26 Leamy M J. On an exact mapping and higher-order Born Rule for use in analyzing graphene carbon nanotubes. Proceedings of the 11th Annual ARL-USMA Technical Symposium, November 5, 2003

27 Xiao S P, Yang W X. Temperature-related Cauchy-Born rule for multiscale modeling of crystalline solids. Comput Mater Sci, 2005,

28 Ericksen J L. The Cauchy-Born hypothesis for crystals. In: Gurtin M, ed. Phase Transformations and Material Instabilities in Solids. New York: Academic Press, 1984. 50-66

29 Arroyo M, Belytschko T. An atomistic-based finite deformation membrane for single layer crystalline films. J Mech Phys Solids, 2002, 50: $1941-1977$

30 Bažant Z P, Gambarova P G. Crack shear in concrete: Crack band microplane model. J Struct Eng, 1984, 110: 2015-2036

31 Bažant Z P, Pijaudier-Cabot G. Nonlocal damage, localization instability and convergence. J Appl Mech, 1988, 55: 287-293

32 Bažant Z P, Prat P. Microplane model for brittle plastic material, I. Theory and II. Verification. J Eng Mech, 1988, 114: 1672-1702

33 Kuhl E, Ramm E, Borst R D. An anisotropic gradient damage model for quasi-brittle materials. Comput Meth Appl Mech Eng, 2000, 183: $87-103$

34 Que N S, Tin-Loi F. Numerical evaluation of cohesive fracture parameters from a wedge splitting test. Eng Fract Mech, 2002, 69: $1269-1286$

35 Planas J, Elices M. Nonlinear fracture of cohesive materials. Int J Fract, 1991, 51: 139-157

36 Bolzon G, Ghilotti D, Maier G. Parameter identification of the cohesive crack model. In: Sol H, Oomens C W J, eds. Material Identification Using Mixed Numerical and Experimental Methods. Dordredt: Kluwer, 1997. 213-222

37 Tin Loi F, Li H. Numerical simulations of quasibrittle fracture processes using the discrete cohesive crack model. Int J Mech Sci, 2000, 42: $367-379$

38 Belytschko T, Organ D, Gerlach C. Element-free Galerkin methods for dynamic fracture in concrete. Comput Meth Appl Mech Eng, 2000, 187: $385-399$

39 Borst R D. Numerical aspects of cohesive-zone models. Eng Fract Mech, 2003, 70: 1743-1757

40 Needleman A. Material rate dependence and mesh sensitivity in lo- 
callization problems. Comput Meth Appl Mech Eng, 1988, 67: 6985

41 Wells G N, Sluys L J. Three-dimensional embedded discontinuity model for brittle fracture. Int J Solids Struct, 2001, 38: 897-913

42 Lasry D, Belytschko T. Localization limiters in transient problems. Int J Solids Struct, 1988, 24: 581-597

43 Peerlings R H J, Borst R D, Brekelmans W A M, et al. Localization issues in local and nonlocal continuum approaches to fracture. Eur $\mathrm{J}$ Mech A-Solids, 2002, 21: 175-189

44 Peerlings R H J, Geers M G D, Borst R D, et al. A critical comparison of nonlocal and gradient-enhanced softening continua. Int J Solids Struct, 2001, 38: $7723-7746$

45 Chang C S, Askes H, Sluys L J. Higher-order strain/higher-order stress gradient models derived from a discrete microstructure, with application to fracture. Eng Fract Mech, 2002, 69: 1907-1924

46 Chen J S, Zhang X W, Belytschko T. An implicit gradient model by a reproducing kernel strain regularization in strain localization problems. Comput Meth Appl Mech Eng, 2004, 193: 2827-2844

47 Peerlings R H J, Borst R D, Brekelmans W A M, et al. Grading enhanced damage for quasi-brittle materials. Int J Numer Methods Eng, 1996, 39: 3391-3403

48 Simone A, Wells G N, Sluys L J. From continuous to discontinuous failure in a gradient-enhanced continuum damage model. Comput Meth Appl Mech Eng, 2003, 192: 4581-4607

49 Cosserat E, Cosserat F. Theory Des Corps Deformables. Paris: Herman et Fils, 1909

5050 Eringen A C. Microcontinuum Field Theories I: Foundations and Solids. New York: Springer, 1999 